\title{
Statistics of rare strong bursts in autocatalytic stochastic growth with diffusion
}

\author{
$\operatorname{AUTHOR}(S)$ :
}

Nakao, H; Mikhailov, AS

\section{CITATION:}

Nakao, $\mathrm{H}$... [et al]. Statistics of rare strong bursts in autocatalytic stochastic growth with diffusion. CHAOS 2003, 13(3): 953-961

\section{ISSUE DATE:}

2003-09

URL:

http://hdl.handle.net/2433/49981

\section{RIGHT:}

Copyright 2003 American Institute of Physics. This article may be downloaded for personal use only. Any other use requires prior permission of the author and the American Institute of Physics. 


\title{
Statistics of rare strong bursts in autocatalytic stochastic growth with diffusion
}

\author{
Hiroya Nakao \\ Department of Physics, Kyoto University, Kyoto 606-8502, Japan
}

\begin{abstract}
Alexander S. Mikhailov ${ }^{\mathrm{a})}$
Abteilung Physikalische Chemie, Fritz-Haber-Institut der Max-Planck-Gesellschaft, Faradayweg 4-6, 14195 Berlin, Germany
\end{abstract}

(Received 26 February 2003; accepted 6 June 2003; published 22 August 2003)

\begin{abstract}
A general model of autocatalytic stochastic growth with diffusion is analytically and numerically investigated. Exact analytical results for the intermittency exponents and the probability of rare strong bursts in an infinite system are presented. Finite-size saturation effects, leading to the stretched exponential growth of statistical moments, are further considered. These analytical predictions are checked in numerical simulations. () 2003 American Institute of Physics. [DOI: $10.1063 / 1.1596576]$
\end{abstract}

Itinerancy is a characteristic property not only of chaotic, but also of stochastic systems where fluctuations are induced by external noises. These external noises are usually Gaussian because they result from the combined action of many independent random forces. Nonetheless, stochastic response of a system may show large deviations from the Gaussian statistics. In strong rare fluctuations, it would visit regions of its phase space which are not accessible for typical trajectories. Such stochastic dynamics can be viewed as an alteration of rare strong bursts and long periods with moderate fluctuations. The origin of stochastic itinerancy (also known as intermittency) can be traced to the presence of repellers. Whenever a phase trajectory passes close to a repeller, it becomes sensitive to noise and strong fluctuations are thus developing. This behavior is especially interesting for spatially extended systems. The presence of repellers is a common property of chaotic and stochastic itinerancies. In our contribution, we provide a detailed analysis of a model that serves as a paradigm for stochastic itinerancy in extended systems with local diffusive coupling and is related to synchronization phenomena in cross-coupled lattices of chaotic maps.

\section{INTRODUCTION}

The process of autocatalytic stochastic growth with diffusion is described by

$$
\frac{\partial z}{\partial t}=[\alpha+\eta(x, t)] z+D \frac{\partial^{2} z}{\partial x^{2}},
$$

where the parameter $\alpha$ is the mean growth rate of the real field $z(x, t), D$ is its diffusion constant, and $\eta(x, t)$ is a Gaussian white noise of intensity $s$ with $\langle\eta(x, t)\rangle=0$ and the autocorrelation function

\footnotetext{
a)Electronic mail: mikhailov@fhi-berlin.mpg.de
}

$$
\left\langle\eta(x, t) \eta\left(x^{\prime}, t^{\prime}\right)\right\rangle=2 s \delta\left(t-t^{\prime}\right) \delta\left(x-x^{\prime}\right) .
$$

We interpret this stochastic equation in the Ito sense and consider only the one-dimensional case. The Stratonovich interpretation of Eq. (1) is briefly discussed in Appendix A.

The model (1) defines a dynamical system with a simple structure. In absence of noise, it has a linear repeller at $z$ $=0$ for each spatial point $x$. Local diffusive coupling between such repellers is present. The noise is applied in a multiplicative way, so that it randomly modulates local expansion rates. Note that the model does not include any nonlinearities.

Because of its general form, the model (1) is found in many different applications. Autocatalytic stochastic growth with diffusion typically arises when spatially distributed chemical reactions or reproduction of biological species under random fluctuation of reaction or reproduction rates are considered. $^{1,2}$ The repeller structure is characteristic for activator-inhibitor reaction-diffusion systems where the rate of autocatalytic growth of an activator species can be fluctuating in space and in time. ${ }^{3}$ By a nonlinear transformation of variables, the model (1) can be expressed as the KardarParisi-Zhang (KPZ) equation for stochastic growth of crystals. ${ }^{4}$ A variant of the stochastic equation (1) with a vector field $z$ has been used to describe the phenomenon of generation of strong magnetic astrophysical fields, known as "magnetic dynamo." "5,6 A similar equation describes the development of material structures in the early inflationary stage of the Universe. ${ }^{7}$ On the other hand, it also arises when synchronization in two coupled arrays of chaotic maps is considered. ${ }^{8}$ Finally, statistical cluster analysis of random fields generated by this equation shows strong similarities with the intermittency observed in hydrodynamic turbulence. ${ }^{9}$

Though "chaotic itinerancy," which is the subject of this Focus Issue, has rather broad meaning, it typically arises as chaotic hopping between various weak attractors or saddles embedded in a high-dimensional phase space. For example, Kaneko showed that chaotic itinerancy observed in globally 
coupled chaotic maps can be associated with the existence of a network of multiple fragile (Milnor) attractors. ${ }^{10}$ Although his model had many degrees of freedom and unraveling of its complex phase space structure is still an unsolved challenging problem, it was pointed out (see, e.g., Ref. 11) that the behavior of an orbit in the vicinity of one of such attractors shares common features with the so-called "on-off intermittency," which can readily be observed in low-dimensional dynamical systems.

The on-off intermittency was first reported by Fujisaka and Yamada in a system of two coupled chaotic maps. ${ }^{12}$ Due to the symmetry, the system had an invariant manifold in which the synchronized chaotic attractor was embedded. ${ }^{13}$ When the coupling strength was slightly less than the threshold for chaotic synchronization, the difference of the variables of the two maps exhibited strong intermittent bursting. This was a consequence of creation of "holes" on the invariant manifold, resulting in occasional long excursions of the orbit escaping from the synchronized chaotic attractor through such holes. Such intermittent dynamics of orbits can be described by a single linear multiplicative stochastic process, which well reproduces some of its statistical properties. ${ }^{13}$

Recently, cross-coupled spatiotemporally chaotic systems have received much attention. ${ }^{14,15}$ Considering synchronization in two coupled map lattices, it was pointed out ${ }^{8}$ that the linearized field of the difference between the local states of the two coupled map lattices can be described by a multivariate multiplicative stochastic process with diffusion, i.e., by a discrete version of the stochastic autocatalytic system (1). The desynchronization transition in cross-coupled chaotic map lattices can be regarded as a variant of spatiotemporal on-off intermittency. Therefore, a detailed analysis of the stochastic autocatalytic system (1) is also important for better understanding of synchronization phenomena for spatiotemporal chaos.

A remarkable property of the stochastic equation (1) is that it is exactly solvable. . $^{2,16,17}$ The evolution of statistical moments of the field $z(x, t)$ is governed by the same linear operator as the Hamiltonian for a system of quantum identical particles interacting via a contact binary attractive potential, whose exact spectrum is known. ${ }^{18}$ The variational equations for optimal fluctuations, corresponding to the model (1), are identical (up to a certain transformation) to the nonlinear Schrödinger equation (NSE) which is exactly integrable using the inverse scattering methods. ${ }^{19,20}$ Note that the NSE is, in turn, a classical limit for the quantum Schrödinger equation describing a system of identical particles with attractive binary interactions.

Figure 1 shows a characteristic spatial distribution of the field $z$ generated by the process of autocatalytic stochastic growth with diffusion (1). It contains a number of bursts of varying strengths. Such bursts can be viewed as large local excursions from the low-density quiescent state. The aim of this paper is to systematically discuss the statistics of such rare strong bursts in model (1). In Sec. II, exact results for an infinite system are presented. Section III is devoted to approximate analytical estimates for finite-size saturation effects in this model. Numerical simulations of the

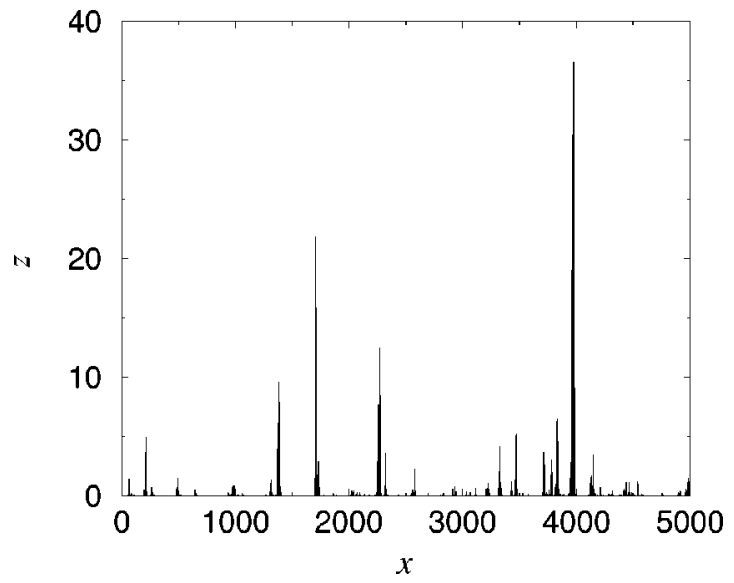

FIG. 1. Typical snapshot of the field $z(x, t)$. The system size is $N=5000$.

stochastic differential equation (1) are reported in Sec. IV. The paper ends with the discussion of obtained results. In Appendix A the Stratonovich interpretation of the considered stochastic differential equation is briefly discussed. The algorithm, used for numerical integration of Eq. (1), is presented in Appendix B.

\section{EXACT ANALYTICAL RESULTS FOR AN INFINITE SYSTEM}

The stochastic model (1), describing autocatalytic growth with diffusion, is linear and its exact analytical solutions can be constructed. Such exact results for an infinitely extended system are presented in this section.

\section{A. Intermittency exponents}

In order to characterize the growth property of the field, we focus on the growth rates of various statistical moments of the field variable. Let us denote the $k$ th statistical moment of the field $z(x, t)$ by

$$
M_{k}(t)=\left\langle z(x, t)^{k}\right\rangle .
$$

Here and in the following all averages are taken with respect to an ensemble of different realizations of the stochastic process $z(x, t)$. We also consider relative statistical moments, i.e., the ratios of the $k$ th moment to the $k$ th power of the first moment $M_{k}(t) / M_{1}(t)^{k}$, and define the intermittency exponents $\Lambda_{k}$ as

$$
\Lambda_{k}=\lim _{t \rightarrow \infty} \frac{1}{t} \ln \left[\frac{M_{k}(t)}{M_{1}(t)^{k}}\right] .
$$

If $\Lambda_{k}$ is nonvanishing, the relative moments grow exponentially with time. It means that the evolution of the probability density of $z(x, t)$ is not self-similar.

In absence of diffusion $(D=0)$, Eq. (1) reduces to a simple Langevin equation

$$
\frac{\partial z}{\partial t}=[\alpha+\eta(t)] z
$$

By solving the Fokker-Planck equation describing the probability density function of the variable $z(t)$, it can be shown ${ }^{2}$ that $M_{k}(t)$ grows with time as 


$$
M_{k}(t) \sim \exp \left[k \alpha t+\left(k^{2}-k\right) s t\right],
$$

and the intermittency exponent for $D=0$ is

$$
\Lambda_{k}=\left(k^{2}-k\right) s \text {. }
$$

The growth rate for system (1) with diffusion has been earlier determined by A.S.M. (see Refs. 2, 16, and 17). The statistical moment $M_{k}=\left\langle z(x, t)^{k}\right\rangle$ for this system obeys the following evolution equation:

$$
\dot{M}_{k}=k \alpha M_{k}-\hat{L}_{k} M_{k},
$$

where the linear self-adjoint differential operator $\hat{L}_{k}$ is given by

$$
\hat{L}_{k}=-D \sum_{i=1}^{k} \frac{\partial^{2}}{\partial x_{i}^{2}}-s \sum_{i, j=1, i \neq j}^{k} \delta\left(x_{i}-x_{j}\right) .
$$

The long-time asymptotics of the $k$ th statistical moment for $t \rightarrow \infty$ is therefore determined by the lowest eigenvalue $\mu_{k}$ of this operator as

$$
M_{k}(t) \sim \exp \left[\left(k \alpha-\mu_{k}\right) t\right] .
$$

The linear operator $\hat{L}_{k}$ is identical to a Hamiltonian for a system of $k$ quantum particles interacting via a binary attractive potential $u(x)=-s \delta(x)$. The exact energy levels (that is, the eigenvalues of $\hat{L}_{k}$ ) for this quantum system are known. ${ }^{18}$ Different energy states correspond to various partitions of $k$ particles into groups, each forming a certain bound state. The deepest energy level corresponds to a bound state of all $k$ particles and the respective eigenvalue for an infinite domain $x \in(-\infty, \infty)$ is ${ }^{18}$

$$
\mu_{k}=-\frac{1}{12} \frac{s^{2}}{D}\left(k^{3}-k\right) \text {. }
$$

Hence, the asymptotic behavior of the moments is given by

$$
M_{k}(t) \sim \exp \left[k \alpha t+\frac{s^{2}}{12 D}\left(k^{3}-k\right) t\right],
$$

and the exact intermittency exponent for the autocatalytic stochastic growth with diffusion is ${ }^{16}$

$$
\Lambda_{k}=\frac{1}{12} \frac{s^{2}}{D}\left(k^{3}-k\right) \text {. }
$$

By comparing the intermittency exponents (7) and (13), we see that autocatalytic growth with diffusion shows even higher deviations from the Gaussian statistics than the respective process in absence of diffusion.

The growth law (12) holds for the Ito interpretation of the stochastic differential equation (1). In the Stratonovich interpretation, the coefficient $\alpha$ is additionally renormalized by noise (see Ref. 16). However, the intermittency exponents are the same for both interpretations and are always given by Eq. (13).

\section{B. Statistics of bursts}

A typical realization of the field $z(x, t)$ includes bursts separated by long intervals of low activity (cf. Fig. 1). Each burst can be viewed as a rare strong fluctuation of the stochastic field $z(x, t)$. The exact statistics of rare strong bursts for autocatalytic growth with diffusion has previously been determined. ${ }^{2,16,17}$ Introducing the functional $P[z(x, t)]$ which gives the probability density for various statistical realizations of the field $z(x, t)$, a solution for this functional in the form of a path integral is obtained as

$$
P[z(x, t)]=\int \mathcal{D} \rho(x, t) \exp \left[-\int d t\left(-H+\int d x \dot{z} \rho\right)\right],
$$

where $\rho(x, t)$ is the auxiliary real field and

$$
H=\int d x\left(D \rho \frac{\partial^{2} z}{\partial x^{2}}+s \rho^{2} z^{2}\right)
$$

For simplicity we take $\alpha=0$ here and in the following.

The optimal trajectories for the considered random process correspond to the minima of "action"

$$
S=\int d t\left(-H+\int d x \dot{z} \rho\right)
$$

and obey variational equations

$$
\dot{z}=\frac{\delta H}{\delta \rho(x, t)}, \quad \dot{\rho}=-\frac{\delta H}{\delta z(x, t)},
$$

or, explicitly,

$$
\begin{aligned}
& \dot{z}=2 s \rho z^{2}+D \frac{\partial^{2} z}{\partial x^{2}}, \\
& \dot{\rho}=-2 s z \rho^{2}-D \frac{\partial^{2} \rho}{\partial x^{2}} .
\end{aligned}
$$

The probability $p$ of an exponentially rare fluctuation can be estimated by finding the respective solution of these variational equations, determining the corresponding value of the action $S$, and taking in the saddle-point approximation $p$ $\sim \exp (-S)$.

The variational equations (18) and (19) are effectively equivalent to the nonlinear Schrödinger equation

$$
i \dot{\psi}=-2 \psi^{2} \psi^{*}-\frac{\partial^{2} \psi}{\partial x^{2}}
$$

for the complex field $\psi(x, t)$. To establish this correspondence, we additionally write the nonlinear Schrödinger equation for the complex conjugate function $\psi^{*}$,

$$
i \dot{\psi}^{*}=2 \psi^{* 2} \psi+\frac{\partial^{2} \psi^{*}}{\partial x^{2}} .
$$

Variational equations (18) and (19) are obtained from Eqs. (20) and (21), if we formally treat $\psi$ and $\psi^{*}$ as two independent variables and perform transformations $t \rightarrow-i t, \psi \rightarrow z$, and $\psi^{*} \rightarrow s \rho$. Additionally, the coordinate $x$ should be appropriately rescaled.

Because the nonlinear Schrödinger equation (NSE) is completely integrable, the same holds for the variational equations (18) and (19). Each soliton solution of the NSE generates a certain solution of the variational equations, which can be obtained by applying the above-given transformations. The NSE solitons form a two-parameter family, and 
are defined by their width and velocity. Immobile NSE solitons (with the zero velocity) give rise to the following solutions of variational equations: ${ }^{16}$

$$
z_{q}(x, t)=\frac{q^{1 / 2} \exp (q t)}{\cosh \left[(q / D)^{1 / 2}\left(x-x_{0}\right)\right]} .
$$

The validity of such solutions can be verified by direct substitution. We see that they represent bursts localized at an arbitrary point $x_{0}$ in the coordinate space and growing exponentially with time. Such burst solutions depend on a free parameter $q$ that specifies the width of a burst and, at the same time, determines its rate of exponential growth.

Generally, variational equations for a certain stochastic process describe optimal fluctuations, i.e., the most probable realizations of a stochastic process satisfying certain constraints (such as, e.g., initial conditions). Localized bursts (22) yield the most probable realizations of the stochastic field $z(x, t)$ that exponentially grow at any given rate $q$. If such realizations are exponentially rare, their probability can be evaluated by taking burst solutions (22) of the variational equations and determining the action $S$ for such solutions. Thus, we find that the probability $p(q, T)$ to find a strong burst with a growth rate $q$ existing within a time interval $T$ is given $b^{2,16}$

$$
p(q, T)=C(q, T) \exp \left(-\frac{4 q^{3 / 2} D^{1 / 2} T}{3 s}\right),
$$

where $C(q, T)$ is a preexponential factor which remains unknown.

In addition to standing bursts which are described by Eq. (22), traveling bursts corresponding to traveling NSE solitons are possible. Such traveling burst solutions have been constructed and analyzed. ${ }^{2,16}$ They are similar to the burst solutions (22) and also exponentially grow with time, while moving in a certain direction. The probability of such traveling bursts, growing at a rate $q$ and traveling at velocity $V$, can also be estimated. ${ }^{2,16}$ It is, however, less than that for the standing bursts with the same rate of growth. Therefore, the statistics is dominated by standing bursts.

If we define a function $f(q)$ through

$$
p(q, T)=C(q, T) \exp (-T f(q))
$$

it is given, according to Eq. (23), by

$$
f(q)=\frac{4 D^{1 / 2}}{3 s} q^{3 / 2} \text {. }
$$

Note that only the probability of exponentially rare fluctuations can be estimated using the saddle-point approximation for the path-integral solution. This implies that the product $T f(q)$ in the exponent in the distribution (24) should be large, $T f(q) \gg 1$.

A similar analysis can be performed for the system without diffusion $(D=0)$. Integrating Eq. (5), we get

$$
z(t+T)=z(t) \exp \left[\int_{t}^{t+T} \eta\left(t^{\prime}\right) d t^{\prime}-s T\right],
$$

where the integral of the stochastic process $\eta(t)$ should be taken according to the Ito interpretation (see Appendix A). The stochastic growth rate over time interval $T$ is then defined as

$$
q=\frac{1}{T} \int_{0}^{T} \eta\left(t^{\prime}\right) d t^{\prime}-s .
$$

As a sum of independent Gaussian noises, $q$ is itself a Gaussian random variable with $\langle q\rangle=-s$ and $\left\langle\Delta q^{2}\right\rangle=2 s / T$. Therefore, its probability distribution is

$$
p(q, T) \sim \exp \left[-\frac{(q+s)^{2} T}{4 s}\right]
$$

and the function $f(q)$ in absence of diffusion is given by

$$
f(q)=\frac{(q+s)^{2}}{4 s} .
$$

Note that, in contrast to Eqs. (23) and (25), these results are not restricted to strong rare bursts.

\section{The Legendre transform method}

The exact asymptotic behavior of all statistical moments $M_{k}(t)$ of a random field $z$ in the limit of long time is already known and given (for $\alpha=0$ ) by $M_{k}(t) \sim \exp \left(\Lambda_{k} t\right)$ with the intermittency exponents (13). Suppose that we can represent such statistical moments as a linear superposition of some components growing exponentially with time, that is as

$$
M_{k}(t)=\int_{0}^{\infty} \exp (k q t) p(q, t) d q,
$$

where $p(q, t)$ can be viewed as statistical weights of such components. Suppose further that such weights are exponentially small and therefore we can write $p(q, t)=C(q, t)$ $\times \exp [-t f(q)]$. Then the integral decomposition (30) takes the form

$$
M_{k}(t)=\int_{0}^{\infty} C(q, t) \exp [(k q-f(q)) t] d q .
$$

As we know, the moments $M_{k}(t)$ grow exponentially with time in the long-time limit. This means that the integral (31) should be dominated at $t \rightarrow \infty$ by a single component with a certain growth rate $q_{k}^{*}$. This value of $q$ is determined by the condition that the exponent in the integrand of (31) has a maximum at $q=q_{k}^{*}$. If the exponent $\lambda_{k}$ for the growth of $k$ th statistical moment is defined as $\lambda_{k}$ $=\lim _{t \rightarrow \infty} t^{-1} \ln M_{k}(t)$, it should be related to the function $f(q)$ by

$$
\lambda_{k}=\max _{q}[k q-f(q)] .
$$

When all exponents $\lambda_{k}$ are known, the function $f(q)$ can therefore be determined by the inverse Legendre transform of $\lambda_{k}$.

In the considered problem of autocatalytic stochastic growth with diffusion, we have $\lambda_{k}=\left(s^{2} / 12 D\right)\left(k^{3}-k\right)$ and thus its inverse Legendre transform yields 


$$
f(q)=\frac{4 D^{1 / 2}}{3 s}\left(q+\frac{s^{2}}{12 D}\right)^{3 / 2} .
$$

It can be verified by direct substitution of function (33) into the integral (31) that this integral indeed yields in the longtime limit the statistical moments $M_{k}(t)$ with the intermittency exponents (13).

The "components," characterized by various growth rates $q$, were formally introduced above through an integral decomposition of statistical moments. For sufficiently high growth rates, these components can however be identified as strong bursts, considered in the previous section. The exponentially rare bursts (22), growing at various rates $q$, are independent and well separated in space. Therefore, the contributions from such rare bursts should also satisfy a decomposition (30), where, however, the weights $p(q, t)$ would already represent probability densities to find a burst with a rate $q$ within time $t$.

Hence, it interesting to compare the functions $f(q)$ yielded by the inverse Legendre transform of the exact intermittency exponents [Eq. (33)] with the respective functions (25) obtained using optimal fluctuation arguments. We see that these two functions coincide for $q \gg s^{2} / D$. This means that, for high enough growth rates $q$, the "components" in the integral decomposition (30) can indeed be identified as corresponding to individual rare bursts.

It can be argued that $q_{0}=s^{2} / D$ represents the characteristic growth rate of a "normal" burst and that "strong" bursts should have the rates $q \gg q_{0}$. Note that, by appropriate rescaling of time, coordinates, and the variable $z$, all parameters in the considered stochastic equation (1) can be set to unity. This means that, without loss of generality, we can take $s=D=1$ in all our results. We see that a "normal" burst is characterized by a unit growth rate $q_{0}=1$, in contrast to the "strong" bursts with $q \gg 1$. We conjecture that the optimal-fluctuation estimate (24) and (25) holds, actually, only for such strong bursts.

As follows from Eq. (32), the growth of the $k$ th statistical moment of the field $z(x, t)$ at $t \rightarrow \infty$ is dominated by the component with $q=q_{k}^{*}$, corresponding to the maximum in Eq. (32). Explicitly, we obtain

$$
q_{k}^{*}=\frac{s^{2}}{12 D}\left(3 k^{2}-1\right) \text {. }
$$

Therefore, the growth of statistical moments of low orders ( $k \sim 1$ ) is dominated by normal bursts with $q \sim q_{0}$, whereas high statistical moments with $k \gg 1$ are determined by exponentially rare strong bursts.

\section{FINITE-SIZE SATURATION EFFECTS}

In the above-presented analysis, we have assumed that the system is always sufficiently large to possess within time $t$ a burst with the growth rate $q_{k}^{*}$ giving a dominant contribution to the growth of the $k$ th statistical moment. But the probability to find such bursts decreases with time as $\exp \left[-f\left(q_{k}^{*}\right) t\right]$ and, starting from some time moment, a system of a finite size would not be large enough to possess such a strong burst. This leads to the saturation of intermittency at sufficiently large times in a finite system.

\section{A. Saturation time}

The mean number of bursts with the growth rate $q$ found in a system of the length $L$ and persisting within time $t$ can be estimated as

$$
N(q, t)=L C_{0}(q, t) \exp [-t f(q)]
$$

where the preexponential factor $C_{0}$ is unknown. If this mean number is large, we will always observe a burst with growth rate $q$ within time $t$ in the system of size $L$, while if it is small, such a burst will practically never be found. The characteristic time $t(q)$, separating these two statistical regimes, is roughly estimated from the condition $N(q, t)=1$ as

$$
t(q)=\frac{\ln L_{\mathrm{eff}}}{f(q)},
$$

where an "effective system size" $L_{\text {eff }}=L C_{0}$ is introduced.

According to our previous analysis, the growth of the $k$ th moment $M_{k}(t)$ should be dominated by a burst with the rate $q_{k}^{*}$. However, in a finite system, such a rare burst cannot be observed when $t>t\left(q_{k}^{*}\right)$. Thus, the characteristic saturation time of $M_{k}(t)$ is given by

$$
t_{\mathrm{sat}}=\frac{\ln L_{\mathrm{eff}}}{f\left(q_{k}^{*}\right)}
$$

or, explicitly by using Eqs. (29) and (33),

$$
\begin{aligned}
& t_{\text {sat }}(k)=\frac{1}{k^{2} s} \ln L_{\text {eff }} \quad(D=0), \\
& t_{\text {sat }}(k)=\frac{6 D}{k^{3} s^{2}} \ln L_{\text {eff }} \quad(D>0) .
\end{aligned}
$$

Therefore, higher order moments shall saturate earlier.

\section{B. Stretched exponential growth after saturation}

For $t>t_{\mathrm{sat}}(k)$, the growth of $M_{k}(t)$ is no longer dominated by the bursts with the rate $q=q_{k}^{*}$. Instead, it is determined by the bursts with the maximum rate $q=q^{\dagger}$ within time $t$ which are possible in a system of length $L$. This rate $q^{\dagger}$ can be found by inverting Eq. (36). It is given by

$$
q^{\dagger}(t)=f^{-1}\left(\frac{\ln L_{\mathrm{eff}}}{t}\right) .
$$

Under saturation conditions, the growth rate of the moment $M_{k}(t)$ in the long-time limit can therefore be approximately estimated as

$$
M_{k}(t) \sim \exp \left[\left\{k q^{\dagger}(t)-f\left(q^{\dagger}(t)\right)\right\} t\right] .
$$
(29)

For the diffusionless case $(D=0)$, we obtain from Eq.

$$
q^{\dagger}(t)=\left(4 s \ln L_{\mathrm{eff}}\right)^{1 / 2} t^{-1 / 2}-s
$$

and

$$
M_{k}(t) \sim \exp \left[-k s t+k\left(4 s \ln L_{\mathrm{eff}}\right)^{1 / 2} t^{1 / 2}-\ln L_{\mathrm{eff}}\right] .
$$


Analyzing this result, we notice that in the saturated regime the growth of statistical moments is characterized by a stretched exponential. The dependence of $\ln M_{k}$ on time contains the terms linear in $t$ and proportional to $t^{1 / 2}$.

In the presence of diffusion $(D>0)$, we obtain from Eq. (33)

$$
q^{\dagger}(t)=-\frac{s^{2}}{12 D}+\left(\frac{3 s \ln L_{\mathrm{eff}}}{4 D^{1 / 2}}\right)^{2 / 3} t^{-2 / 3}
$$

and

$$
M_{k}(t) \sim \exp \left[-\frac{k s^{2} t}{12 D}+k\left(\frac{3 s \ln L_{\text {eff }}}{4 D^{1 / 2}}\right)^{2 / 3} t^{1 / 3}-\ln L_{\text {eff }}\right] .
$$

Thus, stretched exponential growth of statistical moments with a nonlinear $t^{1 / 3}$ growth component in addition to the linear exponential growth is found in this case after the saturation. Note that, according to Eq. (44), the rate $q^{\dagger}$ of the dominant burst decreases with time and, at some time, will approach the growth rate $q_{0}$ of a "normal" burst. Our approximation, based on the assumption that statistical moments are determined by a single strongest burst found in the system of a given size, then becomes invalid. Various "normal" bursts with $q \sim q_{0}$ will provide substantial contributions to statistical moments. Therefore, some deviations from the stretched exponential law (45) with the exponent $1 / 3$ are expected at large times.

Summarizing, we have found that, in a finite system of length $L$, the growth of statistical moments $M_{k}(t)$ obeys the exponential law with the intermittency exponent (7) or (13) characteristic for infinite systems until time $t_{\text {sat }}$. After $t_{\text {sat }}$, finite-size saturation effects become dominant and a stretched exponential law (43) or (45) is instead observed. The normalized statistical moments do not depend on time after saturation and are given by

$$
\frac{M_{k}(t)}{M_{1}(t)^{k}} \sim \exp \left[(k-1) \ln L_{\text {eff }}\right] .
$$

Thus, the intermittency is suppressed in a finite system, and saturated relative moments scale with the system size as $\ln L_{\text {eff }}$.

The estimates in this subsection were obtained by considering probabilities of bursts. Strictly speaking, we should have used only the probability distribution (23) and the respective function (25). Instead, the corrected function (33), yielded by the inverse Legendre transform, has been employed. As we shall see in Sec. IV, this leads to good agreement with numerical simulations.

\section{NUMERICAL SIMULATIONS}

To integrate Eq. (1), we employed the explicit Euler method for stochastic differential equations with the time step $\Delta t$ varying from 0.00001 to 0.01 . The coordinate step was always set at $\Delta x=1.0$, so that the simulated system size $L$ coincided with the number $N$ of grid points. The system sizes in our computations varied from $10^{4}$ to $10^{7}$. We had to consider very large systems because effects of exponentially rare statistical fluctuations were investigated. The computations were therefore performed on a vector computer at the

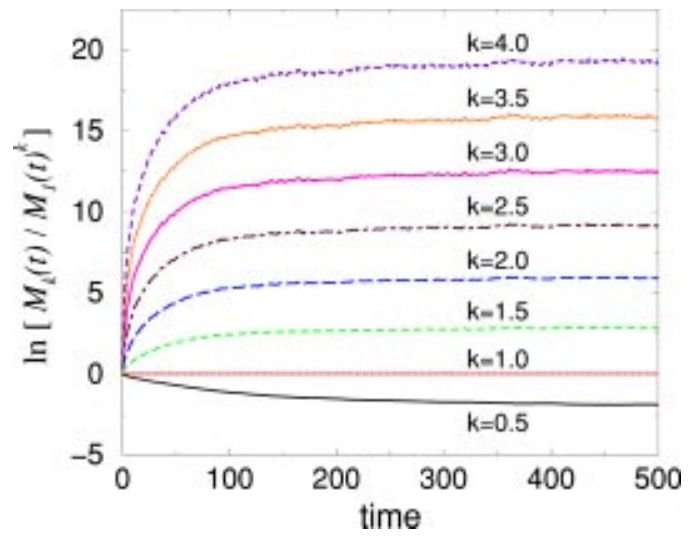

FIG. 2. (Color online) Growth of relative statistical moments $M_{k}(t) / M_{1}(t)^{k}$ of various orders $k$ for a system of size $N=20000$.

Yukawa Institute for Theoretical Physics of Kyoto University. Without loss of generality, the parameters $\alpha$ and $s$ were fixed at $\alpha=0$ and $s=1.0$ in all our simulations; the diffusion constant was always chosen as $D=3.0$. Periodic boundary conditions were used. As the initial condition, a sequence of random numbers from an interval between 0 and 1 has been chosen. Integrations of the stochastic differential equation (1) with such initial conditions and different noise realizations yield an ensemble of fields $\left\{z_{m}(x, t)\right\}$. For each field $m$ in the ensemble, spatial means of $\left[z_{m}(x, t)\right]^{k}$ were first computed at each time moment. Afterwards, these spatial means were additionally averaged over the ensemble of up to 250 different realizations of the field $z(x, t)$ to obtain statistical moments $M_{k}(t)$. Details of the employed numerical algorithm are described in Appendix B.

A typical stochastic realization of the field $z(x, t)$ showing rare strong bursts is presented in Fig. 1. Figure 2 displays the growth of various relative moments in the system of size $N=20000$. We see that initially they increase exponentially, but then the growth becomes saturated. The saturated values of relative statistical moments depend on the moment order $k$. The initial interval with the exponential growth could be extended by running integrations with very large systems. Figure 3 shows in the logarithmic scale the initial growth of

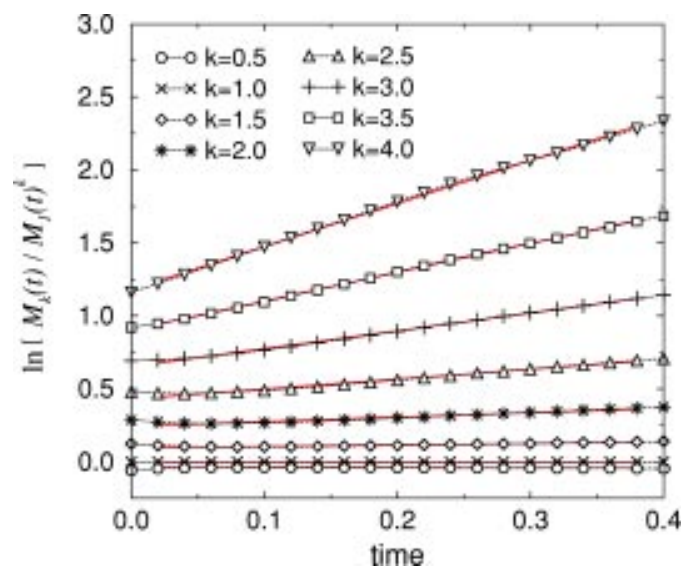

FIG. 3. (Color online) Growth of relative moments $M_{k}(t) / M_{1}(t)^{k}$ of different orders $k$ in the system of size $N=10^{7}$. 


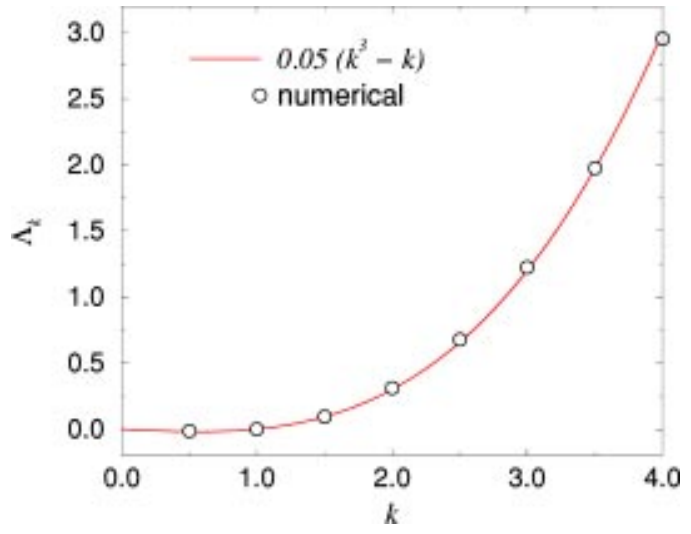

FIG. 4. (Color online) Intermittency exponents $\Lambda_{k}$ for moments of different orders $k$ in the system of size $N=10^{7}$. The solid line shows fitting of the numerical data to the analytical prediction $\Lambda_{k}=A\left(k^{3}-k\right)$ with $A=0.05$.

relative statistical moments of various orders in a much larger system of size $N=10^{7}$. The linear dependence of $\ln \left[M_{k}(t) / M_{1}^{k}(t)\right]$ on time is now evident.

The slopes of the curves, corresponding to different moment orders $k$ in Fig. 3, yield respective intermittency exponents $\Lambda_{k}$. Figure 4 displays these numerically determined intermittency exponents $\Lambda_{k}$ as a function of $k$ (open circles). The solid line in Fig. 4 is obtained by nonlinear least-squares curve fitting to the numerical data. Fitting has been performed by using cubic dependence $A\left(k^{3}-k\right)$ [cf. Eq. (13)] but treating $A$ as an adjustable parameter. A very good agreement with the statistical data has been found for $A=0.05$. Note, however, that this numerical value is substantially larger than the theoretical prediction $A=s^{2} / 12 D \simeq 0.03$. Possible origins of this discrepancy are discussed in Sec. V.

According to Eq. (46), the saturated values of the relative moments $M_{k}(t) / M_{1}(t)^{k}$ should depend linearly both on $(k-1)$ and on $\ln N$. The linear dependence on $(k-1)$ is confirmed numerically in Fig. 5, where saturated values of relative moments for $D=3.0$ are plotted as functions of $k$ for the systems of different sizes. We see that the data points fall on straight lines which cross the horizontal axis at $k=1$, with the slopes depending on the system size $N$. Figure 6 further displays these slopes as a function of the logarithm of the

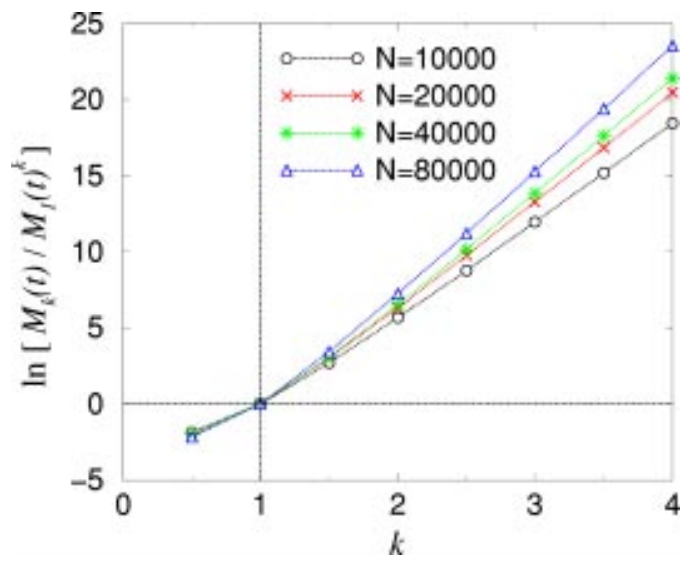

FIG. 5. (Color online) Saturated values of relative statistical moments $M_{k}(t) / M_{1}(t)^{k}$ in systems of different sizes.

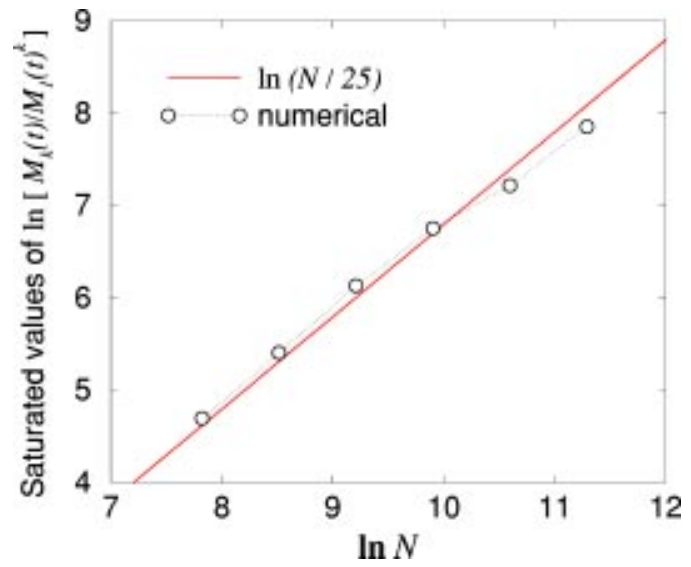

FIG. 6. (Color online) Dependence of saturated values of relative moments $M_{k}(t) / M_{1}(t)^{k}$ on the system size $N$. The logarithmic dependence is apparent; the effective system size $L_{\text {eff }}$ is estimated from this dependence as $L_{\text {eff }}$ $=N / 25$.

system size $N$ (circles). The statistical data can be well fitted by the dependence $\ln (N / C)$ with the fitting parameter $C$ $\simeq 25$ (solid line), which is in agreement with the theoretical predictions. Note that the effective sizes of considered systems were, therefore, $L_{\text {eff }}=N / C$.

In the saturation regime for $t>t_{\mathrm{sat}}$, the growth of statistical moments should be described by the stretched exponential law (45) with the exponent 1/3. According to Eq. (45), the combination

$$
g_{k}(t)=\left[\ln M_{k}(t)-k \beta t+\ln L_{\text {eff }}\right]^{3} / k^{3},
$$

where $\beta=-s^{2} / 12 D$ in the considered case, should increase linearly with time and be independent from $k$. This analytical prediction is numerically tested in Fig. 7 for the system of size $N=20000$. To perform the test, we first notice that, according to Eq. (45), $(1 / t) \ln M_{k}(t)$ should approach a constant equal to $k \beta$ in the long-time limit. In numerical simulations, we find that $(1 / t) \ln M_{k}(t)$ indeed becomes constant at very large times, though the respective constant is a little different from the theoretical prediction. We use the longtime asymptotics of the computed statistical moments to define an effective systematic growth rate $\beta_{\text {eff }}$, which replaces $\beta$ in Eq. (47). Then, we calculate functions $g_{k}(t)$ based on

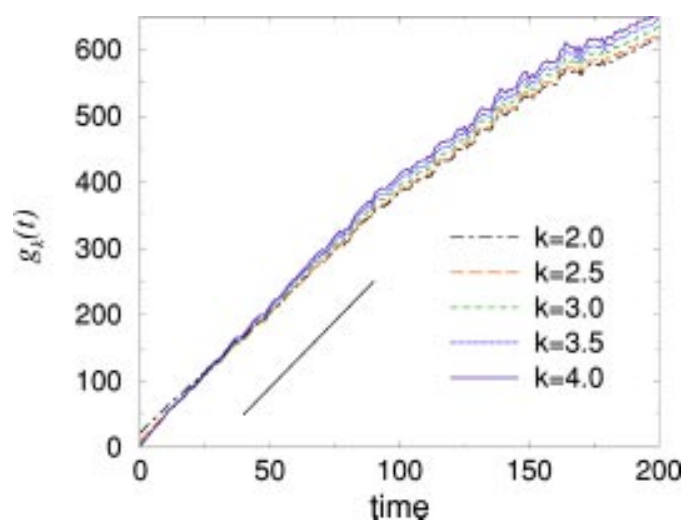

FIG. 7. (Color online) Scaling collapse of statistical moments in system with diffusion. Functions $g_{k}(t)$ for moments of different orders $k$ are displayed for the system of size $N=20000$. 


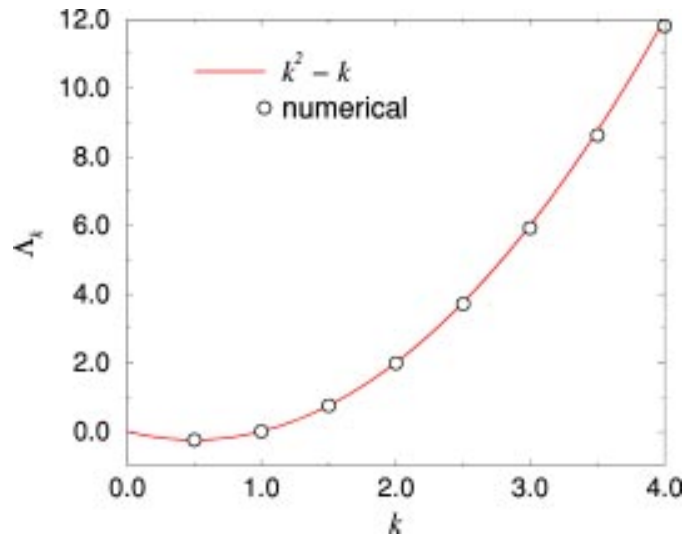

FIG. 8. (Color online) Intermittency exponents $\Lambda_{k}$ for moments of different orders $k$ in the system without diffusion $(D=0)$. The solid line shows the analytical prediction $\Lambda_{k}=k^{2}-k$.

the numerically computed statistical moments: the systematic growth rate is subtracted from the $\operatorname{logarithm} \ln M_{k}(t)$ which is then shifted by $\ln L_{\text {eff }}$. After cubing the result, we find that the curves for different moment orders $k$ collapse after this transformation, as expected in the analytical prediction. A linear dependence on $t$, which confirms the $t^{1 / 3}$ behavior of $\ln M_{k}(t)$, is clearly seen in a broad initial time interval. Subsequently, some deviations from the linear dependence of functions $g_{k}(t)$ develop and the collapse of the curves becomes less pronounced.

Finally, we present results of numerical investigations for the system without diffusion $(D=0)$. Figure 8 shows the computed intermittency exponents for this system (open circles). Full agreement with the analytical prediction (7) is found. Figure 9 presents a test of scaling collapse for the growth of statistical moments. In this case, the saturated growth of statistical moments is described by the stretched exponential law (43) with the exponent of $1 / 2$. According to this law, the combination

$$
h_{k}(t)=\left[\ln M_{k}(t)+k s t+\ln L_{\mathrm{eff}}\right]^{2} / k^{2}
$$

should be independent of the moment order $k$ and should depend linearly on time $t$. Both these properties are confirmed with good accuracy by numerical simulations.

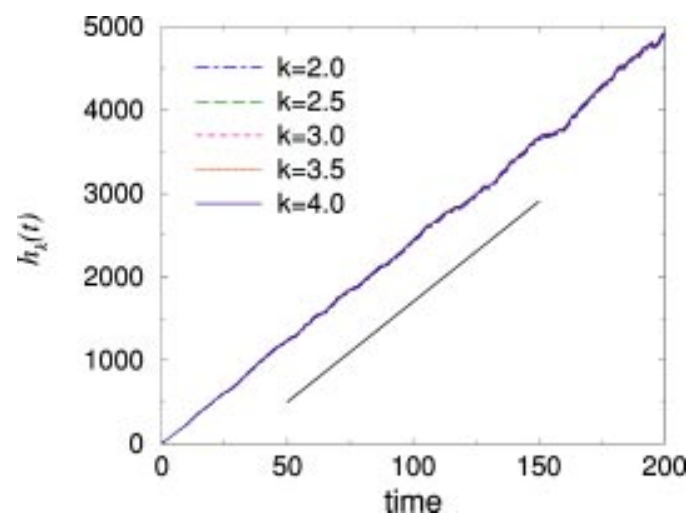

FIG. 9. (Color online) Scaling collapse of statistical moments for the system without diffusion $(D=0)$. Functions $h_{k}(t)$ for moments of different orders $k$ are displayed.

\section{DISCUSSION}

In this article, we have summarized previous exact results for the intermittency exponents and the probability of rare strong bursts, derived new analytical estimates for saturation effects in finite systems, and tested these analytical predictions in numerical simulations. Generally, our numerical tests yield good agreement with the analytical predictions. In the problem of autocatalytic stochastic growth with diffusion, the predicted $k^{3}-k$ dependence of the intermittency exponents is confirmed, and the principal predictions for the saturated growth regime are successfully verified. However, some differences for the linear coefficient $A$ in the intermittency exponents $\Lambda_{k}=A\left(k^{3}-k\right)$ were also found. According to Eq. (13), this coefficient is given by $A=s^{2} / 12 D$ and, thus, should be equal to $A \approx 0.03$ for $s=1$ and $D=3$. In our simulations, the best fit of numerical data has been obtained, however, for $A=0.05$. For the same diffusion constant $D=3$, this value should be analytically expected for somewhat higher noise intensity, $s=\sqrt{5 / 3}=1.291$. Similar systematic discrepancies between exact values and numerical simulations have previously been found and extensively discussed for the Kardar-Parisi-Zhang (KPZ) model. ${ }^{21}$ Their origin apparently lies in the fact that finite-difference schemes, used for the integration of stochastic differential equations with spatially delta-correlated white noise, cannot truly reproduce the fractal nature of their solutions exhibiting strong fluctuations at arbitrarily short length scales. For the KPZ model, the finite-difference simulations yielded numerical values of the diffusion constant which differed from its exact value, but the scaling exponents were, nonetheless, correctly reproduced in these simulations. ${ }^{21}$ Similarly, this numerical difficulty has only a limited effect on our simulations. The numerically obtained intermittency exponents are different from the theoretical prediction, and the agreement could not be improved by going to smaller coordinate steps (mesh steps) $\Delta x$. But once effective renormalization of the noise intensity has been performed, the finite-difference scheme described in Appendix B yields very good agreement with the analytical prediction. The saturation effects at later times, which are not sensitive to the noise intensity, are also correctly reproduced in the simulations.

A comment concerning the relationship between the studied system and the KPZ model should finally be given. The properties of the KPZ model have been actively investigated and many results on the universality class of the KPZ model and its statistical behavior are known. ${ }^{22}$ The reduction of the KPZ model to the problem of autocatalytic stochastic growth involves, however, a nonlinear (logarithmic) transformation of variables. Hence, statistical predictions for the KPZ model cannot be directly transferred to the stochastic equation (1). While being equivalent, the two systems therefore allow one to analyze different facets of stochastic itinerancy (see also Ref. 23).

\section{ACKNOWLEDGMENT}

We acknowledge the assistance of the Yukawa Institute for Theoretical Physics of the Kyoto University in providing the computer facilities. 


\section{APPENDIX A: THE ITO AND STRATONOVICH INTERPRETATIONS}

Any stochastic Langevin equation, including our basic equation (1), can be defined using either the Ito or the Stratonovich interpretations. ${ }^{2,24}$ Roughly speaking, one can say that the Stratonovich interpretation holds when the white noise in a Langevin equation represents a limit of a continuous noise with a vanishingly small, but finite correlation time. On the other hand, the Ito interpretation is applicable when the noise is discontinuous and its values are not correlated at any times. The Ito interpretation is convenient in describing effects of internal noise in chemical reactions, whereas the Stratonovich interpretation is usually employed to study the effects of external fluctuations in electric circuits. Both interpretations correspond to the same FokkerPlanck equation for the probability distribution and there are simple prescriptions, converting one interpretation into another (see Refs. 2 and 24).

The analytical results for the problem of autocatalytic stochastic growth have been presented by us in this article using the Ito interpretation of the stochastic differential equation (1). The respective analysis for the Stratonovich interpretation of this equation has been performed in previous publications. ${ }^{2,16,17}$ The only difference then is that in the Stratonovich interpretation, Eq. (12), describing exponential growth of statistical moments $M_{k}(t)$ in the long-time limit, is modified, i.e., the coefficient $\alpha$ in this equation gets a constant correction proportional to the noise intensity. The intermittency exponents (13) and probability distributions (23) are the same for both interpretations.

In the Stratonovich interpretation, the standard rules of differential calculus are applicable. In contrast to this, special rules should be used in the Ito interpretation when variables are changed or integrals are taken. ${ }^{24}$

\section{APPENDIX B: THE NUMERICAL INTEGRATION ALGORITHM}

Here we explain the numerical integration algorithm used in the simulations. The original Langevin equation is

$$
\frac{\partial z(x, t)}{\partial t}=\alpha z(x, t)+D \frac{\partial^{2} z(x, t)}{\partial x^{2}}+\eta(x, t) z(x, t),
$$

where $\eta(x, t)$ is spatiotemporally white noise,

$$
\begin{aligned}
& \langle\eta(x, t)\rangle=0, \\
& \left\langle\eta(x, t) \eta\left(x^{\prime}, t^{\prime}\right)\right\rangle=2 s \delta\left(x-x^{\prime}\right) \delta\left(t-t^{\prime}\right) .
\end{aligned}
$$

First we spatially discretize

$$
\begin{aligned}
\frac{\partial z_{i}(t)}{\partial t}= & \alpha z_{i}(t)+D \frac{z_{i+1}(t)-2 z_{i}(t)+z_{i-1}(t)}{\Delta x^{2}} \\
& +\eta_{i}(t) z_{i}(t),
\end{aligned}
$$

where we defined $x=i \Delta x$. The correlation of the noise is now given by

$$
\left\langle\eta_{i}(t)\right\rangle=0, \quad\left\langle\eta_{i}(t) \eta_{j}(t)\right\rangle=\frac{2 s}{\Delta x} \delta_{i, j} \delta\left(t-t^{\prime}\right) .
$$

The original equation is thus transformed to a multivariate Langevin equation. We discretize this equation temporally using the explicit Euler scheme:

$$
\begin{aligned}
z_{i}(t+\Delta t)= & z_{i}(t)+\alpha z_{i}(t) \Delta t \\
& +D \frac{z_{i+1}(t)-2 z_{i}(t)+z_{i-1}(t)}{\Delta x^{2}} \Delta t \\
& +z_{i}(t) \sqrt{\frac{2 s \Delta t}{\Delta x}} \xi_{i}(t),
\end{aligned}
$$

where $\xi_{i}(t)$ is a random variable drawn independently from the identical normal Gaussian distribution with mean 0 and variance 1 . Note that the temporal integration given by this numerical scheme corresponds to the Ito integral.

We checked the validity of the above-given discretization scheme using several values for $\Delta x$ (between 0.1 and 1.0 ) and $\Delta t$ (between 0.00001 and 0.01 ) numerically. The statistical moments $\left\langle z(x, t)^{q}\right\rangle$ of the field variable obtained using different values of $\Delta x$ and $\Delta t$ agreed within statistical fluctuation for $q=1,2,3,4$. In this sense, the above-given discretization scheme gives consistent results independent of the mesh size used in the computations, hence the estimated intermittency exponents are also consistent. In the main simulation, we mostly used $\Delta x=1.0$ in order to attain large system size.

${ }^{1}$ A. S. Mikhailov, Phys. Rep. 184, 307 (1989).

${ }^{2}$ A. S. Mikhailov and A. Yu. Loskutov, Foundations of Synergetics. II. Chaos and Noise, 2nd revised and enlarged edition (Springer, Berlin, 1996).

${ }^{3}$ S. Kadar, J. C. Wang, and K. Showalter, Nature (London) 391, 770 (1998).

${ }^{4}$ M. Kardar, G. Parisi, and Y. C. Zhang, Phys. Rev. Lett. 56, 889 (1986).

${ }^{5}$ Ya. B. Zeldovich, S. A. Molchanov, A. A. Ruzmaikin, and D. D. Sokolov, Sov. Phys. Usp. 30, 353 (1987).

${ }^{6}$ Ya. B. Zeldovich, A. A. Ruzmaikin, and A. A. Sokoloff, The Almighty Chance (World Scientific, Singapore, 1990).

${ }^{7}$ A. Linde, Phys. Rep. 333, 575 (2000).

${ }^{8}$ A. Pikovsky and J. Kurths, Phys. Rev. E 49, 898 (1994); A. Pikovsky and A. Politi, Nonlinearity 11, 1049 (1998); V. Ahlers and A. Pikovsky, Phys. Rev. Lett. 88, 254101 (2002).

${ }^{9}$ D. Zanette and A. Mikhailov, Phys. Rev. E 50, 1638 (1994).

${ }^{10}$ K. Kaneko, Physica D 124, 322 (1998).

${ }^{11} \mathrm{P}$. Ashwin, Proceedings of IMA Workshop on pattern formation in coupled and continuous systems, May 1998; P. Ashwin, E. Covas, and R. Tavakol, Nonlinearity 12, 563 (1999).

${ }^{12}$ H. Fujisaka and T. Yamada, Prog. Theor. Phys. 74, 918 (1985).

${ }^{13}$ S. C. Venktaramani, T. M. Antonsen, Jr., E. Ott, and J. C. Sommerer, Physica D 96, 66 (1996).

${ }^{14}$ P. Grassberger, Phys. Rev. E 59, R2520 (1999).

${ }^{15}$ J. Garcia-Ojalvo and R. Roy, Phys. Rev. Lett. 86, 5204 (2001).

${ }^{16}$ A. S. Mikhailov, J. Phys. A 24, L757 (1991).

${ }^{17}$ A. S. Mikhailov, Physica A 188, 367 (1992).

${ }^{18}$ F. A. Berezin, G. P. Pokhil, and V. M. Finkelberg, Vestn. Mosk. Gos. Univ. 1, 21 (1964).

${ }^{19}$ V. E. Zakharov and A. B. Shabat, Sov. Phys. JETP 61, 118 (1971).

${ }^{20}$ L. D. Faddeev, in Solitons, edited by R. K. Bullough and P. J. Caudrey (Springer, Berlin, 1980), p. 339.

${ }^{21}$ C.-H. Lam and F. G. Shin, Phys. Rev. E 57, 6506 (1998).

${ }^{22}$ A.-L. Barabasi and H. E. Stanley, Fractal Concepts in Surface Growth (Cambridge University Press, Cambridge, 1995).

${ }^{23}$ Y. Tu, G. Grinstein, and M. A. Muñoz, Phys. Rev. Lett. 78, 274 (1997); M. A. Muñoz and T. Hwa, Europhys. Lett. 41, 147 (1998).

${ }^{24}$ C. W. Gardiner, Handbook of Stochastic Methods (Springer, Berlin, 1997). 